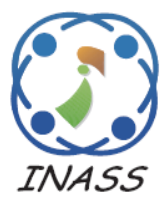

http://www.inass.org/

\title{
A Miniature Microstrip BSF Using Complementary Split Ring Resonator
}

\author{
Badr Nasiri ${ }^{*}$ \\ Ahmed Errkik ${ }^{1}$ \\ Jamal Zbitou ${ }^{1}$ \\ Abdelali Tajmouati ${ }^{1}$ \\ Larbi El Abdellaoui ${ }^{1} \quad$ Mohamed Latrach ${ }^{2}$ \\ ${ }^{1}$ Laboratory of Mechanic, Energetic, Electronic and Telecommunication, Faculty of Sciences and Technics, \\ Hassan first university, Morocco \\ ${ }^{2}$ Microwave Group, Ecole Supérieure d'Electronique de l'Ouest, Angers, France \\ * Corresponding author’s Email: b.nasiri@uhp.ac.ma
}

\begin{abstract}
In this paper, a new microstrip band-stop filter based on a complementary split ring resonator unit cell is proposed, simulated and fabricated on an FR-4 substrate. The design procedure is divided into three sections. Firstly the conventional 3rd Butterworth low-pass prototype a cut-off frequency of $1.2 \mathrm{GHz}$ is designed. Secondly, several series of optimisations have been carried out and studied in order to achieve a miniature circuit size, thirdly the low impedance of the miniaturized LPF is replaced by the chosen resonator with a view to obtaining a band-stop behaviour around of CSRR resonant frequency. The simulated and measured results are compared and good agreement is achieved between them. The proposed band-stop filter is characterized by stopband fractional bandwidth of 58\% with deep rejection can reach $30 \mathrm{~dB}$ at the centre of the rejected band and good electrical performances in the first and second passbands are noticed. This proposed circuit is an adequate solution for the Industrial, Scientific, and Medical band (ISM band) and Universal Mobile Telecommunications System (UMTS IMT-E band).
\end{abstract}

Keywords: Microstrip, Band-stop filter, Metamaterials, Complementary split ring resonator.

\section{Introduction}

Suppression of spurious signals and harmonic interferences produced by the active devices is an essential process in wireless and mobile communication systems [1,2], this operation is performed by the filters. Which mean that the microwave filters are important and indispensable devices in these systems due to their remarkable and outstanding role $[3,4]$.

Recently, the demand for compact and miniature planar components characterized by high- electrical performances has rapidly increased $[5,6]$. That led to exploring various methods and techniques for achieving the microstrip band-stop filters BSFs with excellent features in term of size, band-stop rejection level, and band-pass insertion loss [7, 8]. Among these technics, Defected ground structure DGS [9, 10], electromagnetic band gap EBG but the EBGfilter circuit size remains relatively large which is one of the most major disadvantages to be solved, more particularly when these filters operate at lower frequencies [11], and metamaterials.

Newly, filters based on metamaterials have attracted the attention of scientists and researchers due to their special and unusual characteristics [12, 13]. In 1967, this engineered material was first introduced and theoretically investigated by Veselago [14]. Metamaterial exhibit exceptional and unusual electromagnetic propagation properties are not found in nature and available materials, this artificial structure has a negative permittivity or/and negative permeability in a certain frequency range [15]. After 30 years, Pendry et al proposed the idea to realize a medium have simultaneously negative effective permeability and negative effective permittivity by using Thin-Wires TW and Split Ring Resonators SRRs [16]. The first experimental verification was achieved by smith et al in 2001 . These important theoretical and experimental contribution in electromagnetic field and material properties lead to the beginning of study and development of the metamaterials [17]. That give rise 
to many research works to realize the microwave devices based on metamaterials such as antennas [18], power dividers [19], and filters. However, the circuits were built by using a limited number of resonators and have excellent electrical performances at lower frequencies, they are widely desired.

This letter presents a new microstrip band-stop filter based on complementary split ring resonator which is used to pass from a low pass filter to a bandstop filter. This proposed filter has compact and miniature size. Furthermore, it possesses excellent pass-bands performances and its rejected-band has a Fractional Bandwidth FBW of $58 \%$.

\section{Design procedures}

The classical approaches and conventional technics which are used to design band-stop filter such as filters with open-circuited stubs and optimum band-stop filters generally have a large circuit area especially when these filters operate on the low frequencies. The main objective of this work is to design and develop a novel structure of a compact and miniature band-stop filter has high rejection level in the rejected-band and ensure good transmission in both pass-bands.

The first step in this work is to select the resonator and choose it dimensions to achieve the desired rejected-band and the desired band-stop frequency centre, the second one is to pass from the classical low-pass filter LPF into a miniature stop-band filter BSF based on the selected resonator.

\subsection{Proposed complementary split ring resonator}

The complementary split ring resonator CSRR is one of the famous kinds of metamaterial structures used in the design of various microwave devices such as the antennas, filters, couplers, and power dividers. It has an essential role in the miniaturization and electrical improvement of these components. Unlike the split ring resonator which has a magnetic response, the complementary SSR has electric response and characterized by a negative effective permittivity around of its resonant frequency that can be expressed as follows.

$$
f=\frac{1}{2 \pi \sqrt{L C}}
$$

This frequency can be controlled and shifted by the complementary split ring resonator CSRR dimensions. The Fig. 1 presents the design configurations of the proposed CSRR unit cell. This resonator is designed on an FR4 substrate and its geometry is chosen to obtain a desired resonant

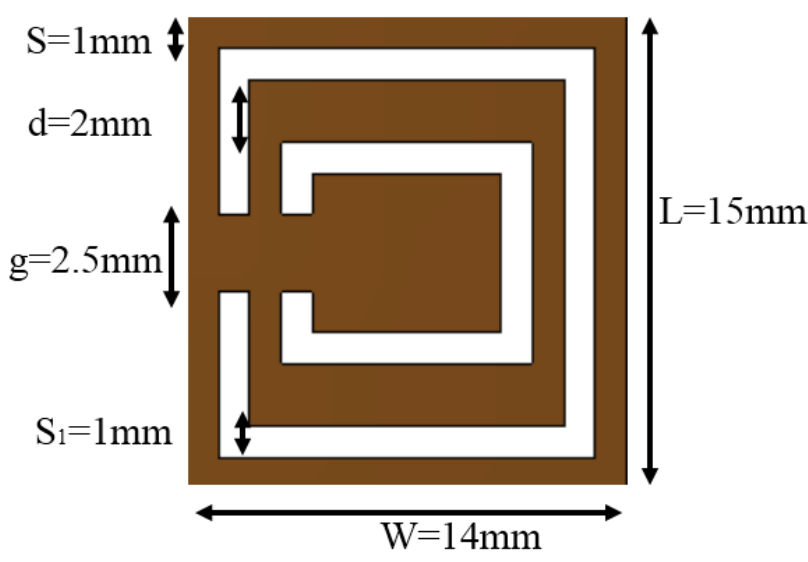

Figure.1 Geometry of the proposed CSRR unit cell

frequency around of $1.6 \mathrm{GHz}$ which will be the rejected-band centre of our proposed band-stop filter.

To verify the existence of the negative permittivity, the complimentary SRR is simulated and the S-parameter Retrieval Method is used to extract the values of the effective medium parameters which are related to the response frequency of the coefficient of transmission $S_{21}$ and reflexion $S_{11}$ by Eq. (2), (3), (4) and (5). $n$ and $z$ present respectively the refractive index the medium and impedance.

$$
\begin{gathered}
n=\frac{1}{k_{\circ} d} \cos ^{-1}\left[\frac{1}{2 S_{21}}\left(1-S_{11}^{2}+S_{21}^{2}\right)\right] \\
z= \pm \sqrt{\frac{\left(1+S_{11}\right)^{2}+S_{21}^{2}}{\left(1-S_{11}\right)^{2}+S_{21}^{2}}} \\
\varepsilon_{\text {eff }}=\frac{n}{z} \\
\mu_{\text {eff }}=n z
\end{gathered}
$$

Where $k_{0}$ presents a wave number equivalent to $2 \pi / \lambda_{0}$, and $d$ is the thickness of the substrate used in the design of the metamaterial resonator.

Fig. 2 illustrates the extracted real and imaginary of the effective permittivity versus frequency of the proposed CSSR unit cell.

\subsection{The proposed band-stop filter}

Firstly, the $3^{\text {rd }}$ Butterworth low-pass prototype is chosen and used as the starting point for our design procedure. Fig. 1, shows its L-C circuit after the frequency transformation from a low-pass prototype to a practical low-pass filter has a cutoff frequency of $1.5 \mathrm{GHz}$ and normalized source impedance $\mathrm{R}_{0}=50$, the calculated L-C elements were obtained using Eq. (6) and (7). Secondly, the series inductors are replaced by high impedances and the shunt capacitor 
is transformed into low impedance transmission section line, the values of physical lengths and widths of each $\mathrm{Z}_{\mathrm{H}}$ and $\mathrm{Z}_{\mathrm{L}}$ are extracted using Eq. (8) and (9). Where $L_{\mathrm{k}}$ and $C_{k}$ present the element values of the $3^{\text {rd }}$ Butterworth low-pass prototype, $R_{0}$ is normalized source impedance that is equal to $50 \Omega$ for practical microstrip filters and $\omega_{\mathrm{c}}$ presents the desired cut-off frequency.

$$
\begin{gathered}
L_{k}^{\prime}=\frac{R_{0} L_{k}}{\omega_{c}} \\
C_{k}^{\prime}=\frac{C_{k}}{\omega_{c} R_{0}} \\
l_{L}=\frac{\omega_{c} L}{Z_{H} \beta} \\
l_{c}=\frac{\omega_{c} C Z_{L}}{\beta}
\end{gathered}
$$

The circuit design of conventional 3rd Butterworth low-pass filter is shown in Fig. 2, and its overall dimensions are listed in Table 1.

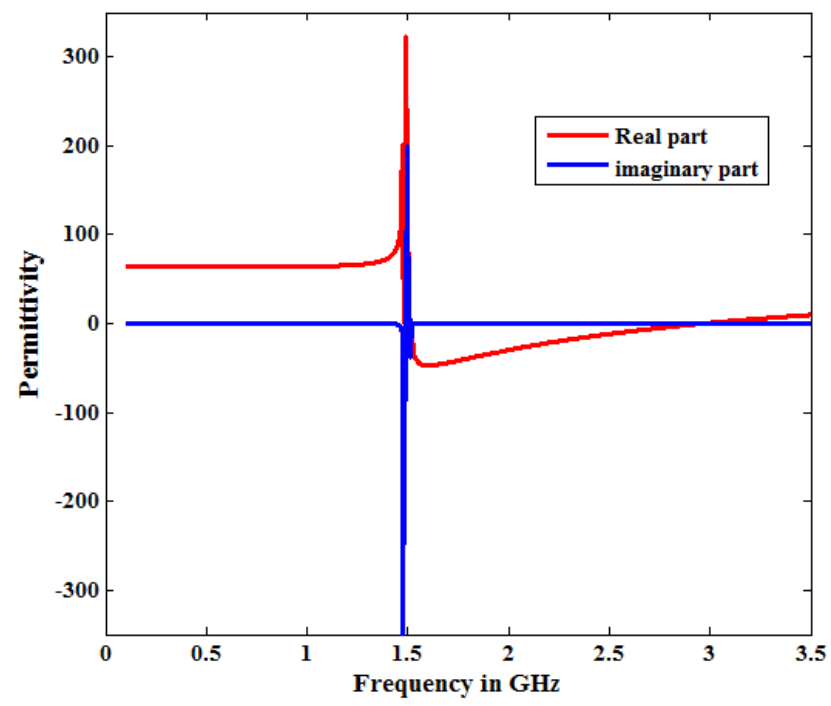

Figure. 2 Real and imaginary of the effective permittivity

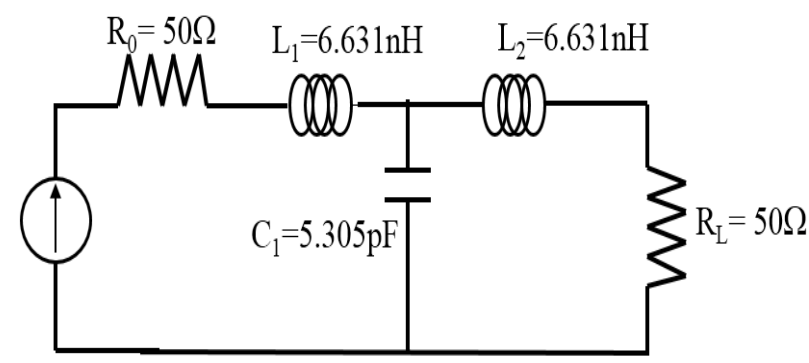

Figure. 3 LC circuit of the LPF
Table 1. Width and length dimensions of LPF

\begin{tabular}{|c|c|c|c|}
\hline $\mathbf{Z}(\mathbf{\Omega})$ & $\mathbf{W}(\mathbf{m m})$ & $\mathbf{L}(\mathbf{m m})$ & $\boldsymbol{\beta I}\left(^{\circ}{ }^{\circ}\right)$ \\
\hline 20 & 11.1169 & 16.3954 & 45,83 \\
\hline 120 & 0.364456 & 9.748960 & 23.860 \\
\hline 50 & 3 & 5 & 13.1 \\
\hline
\end{tabular}

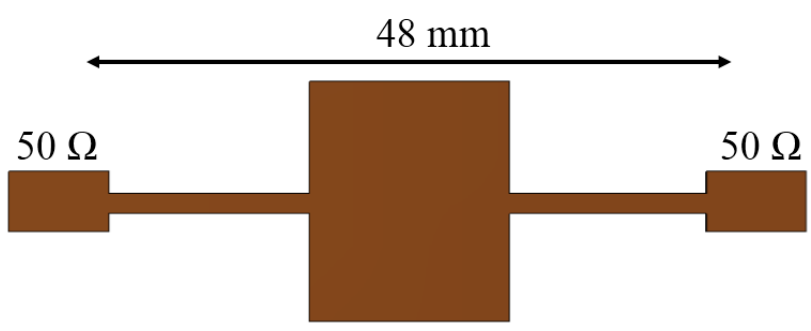

Figure. 4 Layout of the LPF

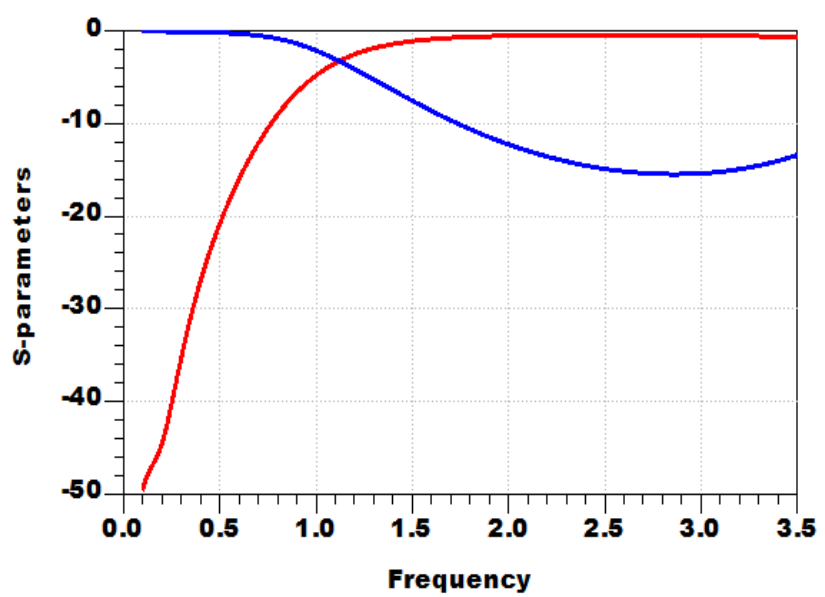

Figure. 5 Frequency response of the conventional LPF

After the validation of the classical stepped impedances LPF that is based on the 3rd Butterworth low-pass prototype. We can pass now to our essential purpose which is to get a novel band-stop filter has excellent features with regard to its overall size and its electrical performances. Without using conventional transformations which used to pass through the classical low-pass filter to a classical band-stop design, because this technic always gives us a large circuit area with modest performances, especially in low range frequencies. Fig.6 presents the classical transformations from low-pass prototype to band-stop filter.

So as to attain these goals. Various series and steps of optimization using different techniques integrated into two electromagnetic solvers (Advanced Design System and CST Microwave Studio) have been carried out and analyzed. Subsequently, the proposed complementary split ring resonator CSRR is etched on top layer of the designed structure in order to reach the stop-band behaviour in undesired band. Fig. 7 presents respectively the designed circuit without the suggested metamaterial 
and Fig. 8 shows the circuit of final proposed bandstop filter.
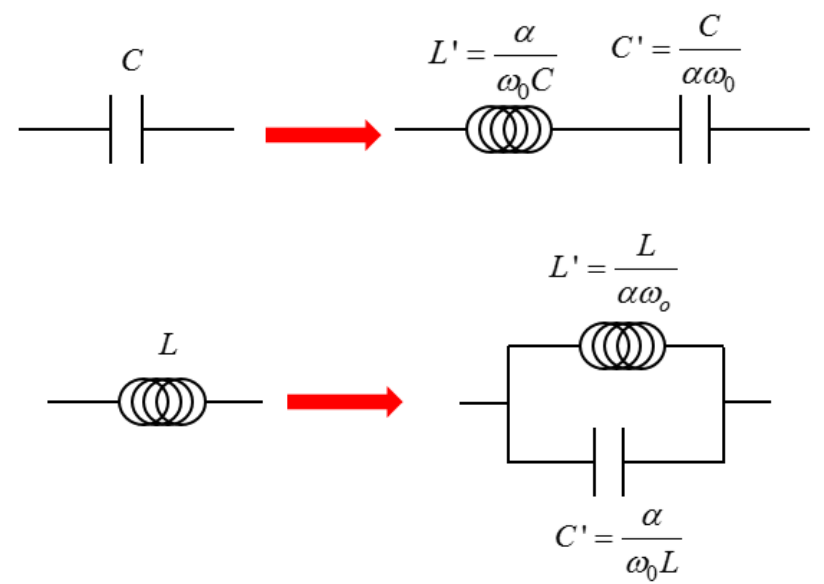

Figure. 6 Classical transformations from LPF to BSF

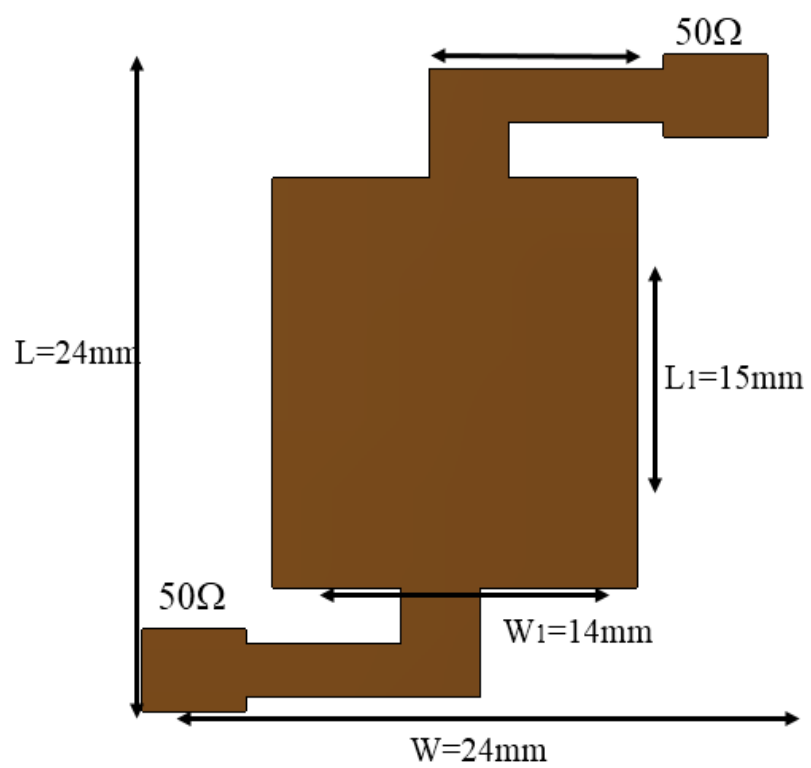

Figure. 7 Miniaturized LPF circuit

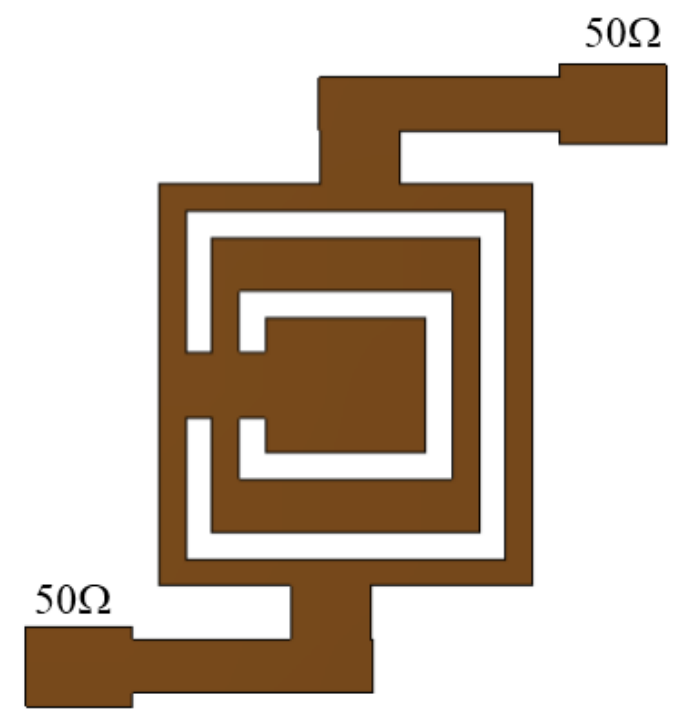

Figure. 8 Proposed BSF circuit

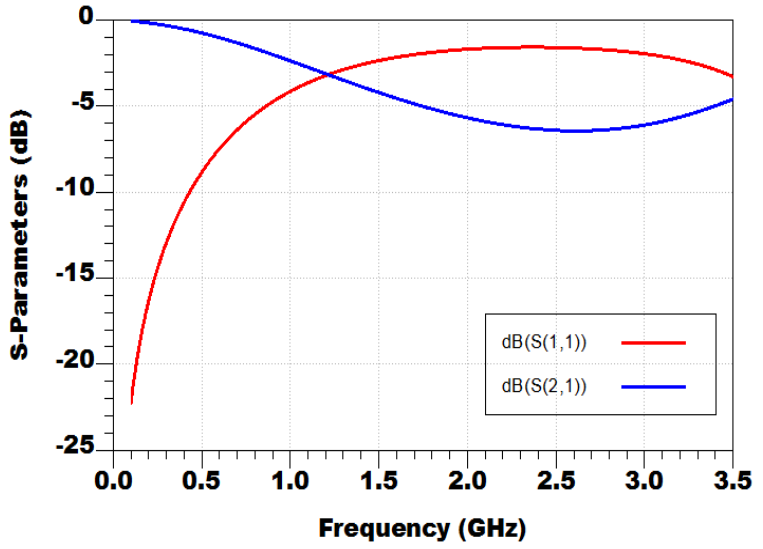

Figure. 9 Frequency responses of the circuit without CSRR

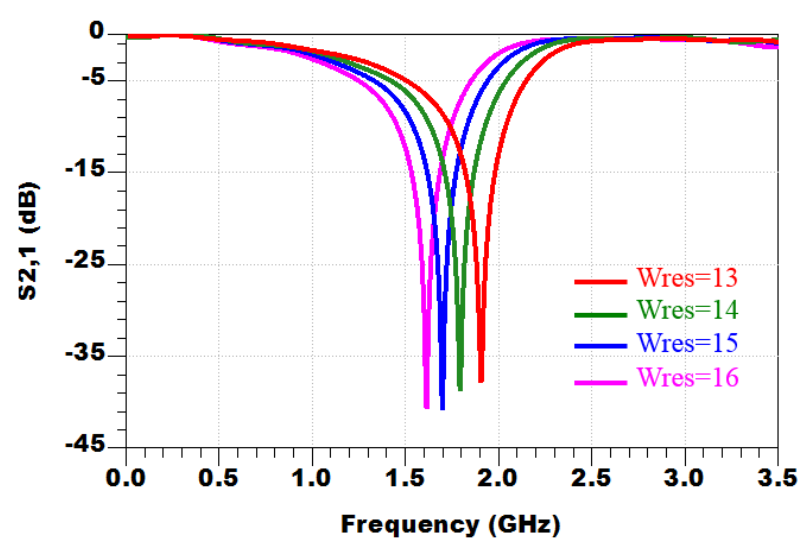

(a)

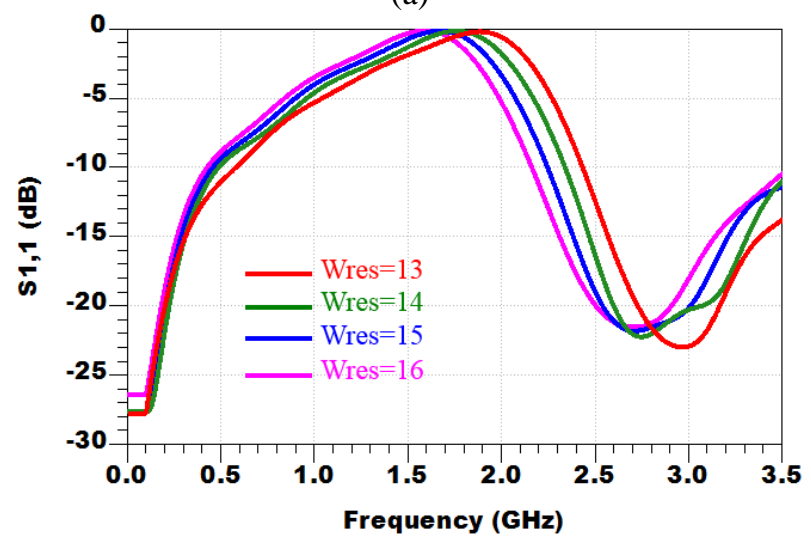

(b)

Figure. 10 (a) Simulated insertion loss for different value of CSRR width and (b) simulated return loss for different value of CSRR width

The miniaturized structure without complementary split ring resonator CSRR metamaterial is simulated at first in order to understand and to investigate the influence and the effect of the proposed resonator unit cell on the designed circuit. Fig. 9 shows its calculated scattering parameters various frequency. As might see, the circuit has low-pass behaviour with cut-off frequency of $1.2 \mathrm{GHz}$. 


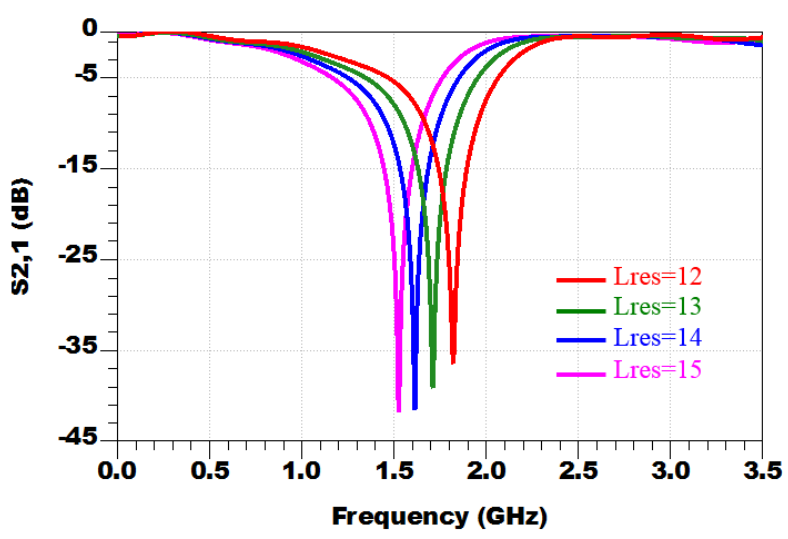

(a)

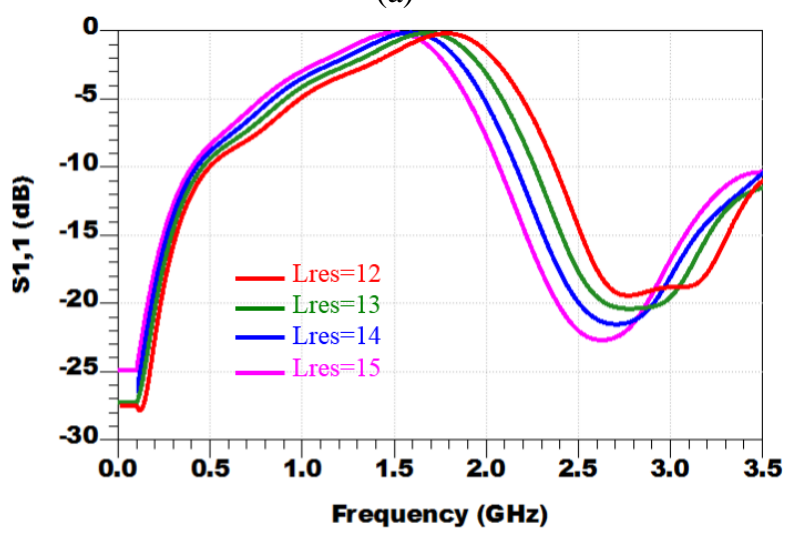

(b)

Figure. 11 (a) Simulated insertion loss for different value of CSRR width and (b) simulated return loss for different value of CSRR width

With a view to investigating the effect and influence of some CSRR parameters on the designed filter. We were started by changing the value of CSRR-width from $13 \mathrm{~mm}$ to $16 \mathrm{~mm}$ and keeping all other parameters remains invariant. As we can see from Fig.10, that as the width is increasing the centre frequency of the undesired band is decreasing, this permits us to say that the transmission zero of the proposed filter can be shifted and controlled by modification of $\mathrm{W}_{\text {res }}$.

Fig. 11 shows the computed S-parameters versus frequency of the distinct CSRR-length which was varied from 12 to 15 in steps of $0.5 \mathrm{~mm}$. As might be seen, the middle of the rejected band can be shifted from $1.9 \mathrm{GHz}$ to $1.6 \mathrm{GHz}$ by increasing the length of the resonator which allows us to say that the centre frequency of the designed band-stop filter can be retrieved by knowing resonator parameters.

The effect of the gap which is located in both rings was investigated by changing the numerical value of the split with all other CSRR-parameters remains fixed. Fig.12 illustrates the calculated scattering parameters against frequency for different values of the gap. It is plain to see, that to achieve a cut-band on the low range frequencies ought to decrease the value of g. Put differently, that as the $g$ is decreased the centre frequency of the stop band is decreased too and shifted to low frequencies.

We can conclude that this proposed filter has a controllable rejected band and its transmission zero can be shifted and located at an undesired frequency by means of complementary split ring resonator parameters.

Fig. 13 describes the S-parameters of the final proposed circuit based on metamaterial unit cell. We can notice that the designed filter shows band-stop characteristic at the centre frequency of $1.7 \mathrm{GHz}$ with a rejected-band extended from $1.2 \mathrm{GHz}$ to $2.2 \mathrm{GHz}$.it is characterized by a deep attenuation can reach more than $30 \mathrm{~dB}$ at $1.7 \mathrm{GHz}$. the first and second passbands have a low insertion loss and an excellent return loss have obtained in both passbands. The simulated scattering parameters show that the filter has FBW of $58 \%$ that is achieved by Eq. (10).

$$
F B W=\frac{f_{2}-f_{1}}{f_{0}} \times 100 \%
$$

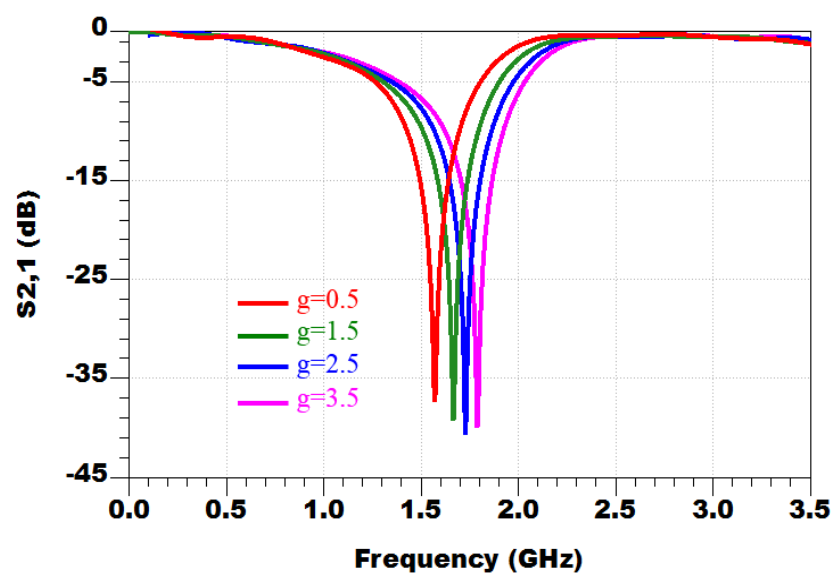

(a)

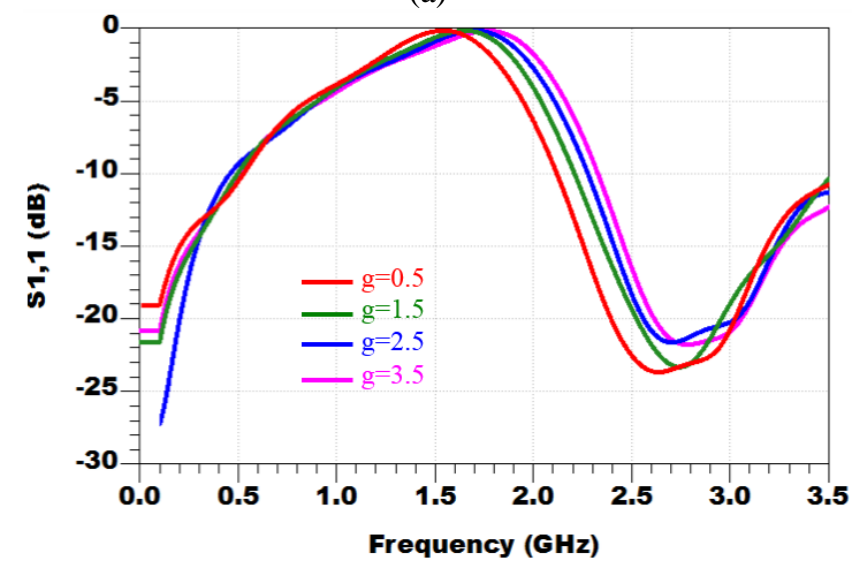

(b)

Figure. 12 (a) Simulated insertion loss for different value of CSRR width and (b) simulated return loss for different value of CSRR width 




(a)

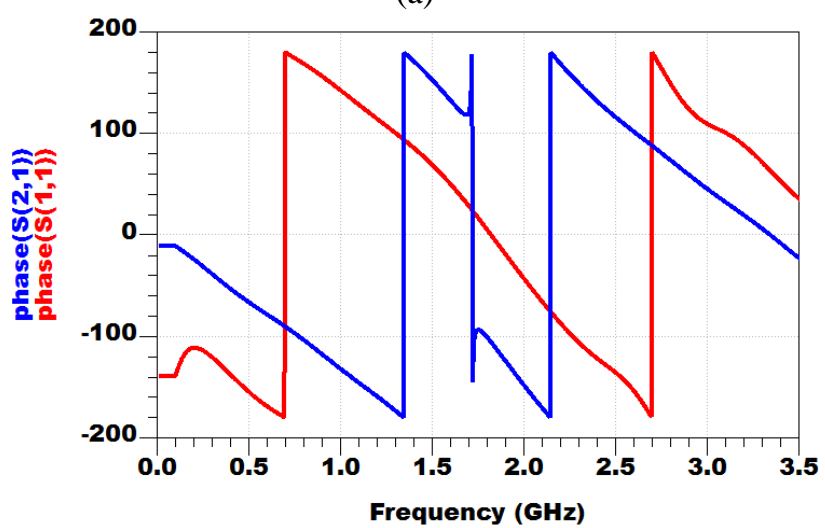

(b)

Figure. 13 (a) Simulated S-parameter magnitude and (b) simulated S-parameters phases

Fig.14 illustrates the simulated surface current in the second pass-band at 2.4.GHz and in the stop-band at $1.6 \mathrm{GHz}$. This short investigation is dedicated to studying the filter electrical performances in the rejected-band and the pass-band state. As we can see, the Fig. 14 (a) confirms that there is no current close to the output port of designed circuit which indicates that there no transmission of RF power from port-1 to port-2.

On the other hand, the surface current on the second selected frequency proves that the signal propagation is transmitted from the input port to output port of the filter. This study authorizes us to say that the designed band-stop filter has good electrical performances in the pass-band state and stop-band state.

\section{Fabrication and measurement}

After various series and steps of optimization using CST Microwave 3D electromagnetic solver. The proposed circuit has been mounted and fabricated on a $1.6 \mathrm{~mm}$ thick FR4 substrate by using LPKF machine and the pair SMA connectors were soldered to the edge of both circuit sides. The manufactured prototype band-stop filter is shown in Fig.15.

The simulated scattering parameters are compared with the measurement results which are obtained by an R\&S Vector Network Analyser HP 8719ES. Fig.16 shows an excellent agreement between the measurement and simulated one. The reflexion and transmission coefficients indicate that the fabricated BSF characterized by stopband fractional bandwidth $\mathrm{FWB}=58 \%$ and a central frequency of $1.7 \mathrm{GHz}$. the circuit provides a highlevel power rejection of undesired signals in the stopband and good return and insertion loss have reached in both passbands. The small deviations between the simulated and measured results are principally produced by an error in the manufacturing or measuring process or dielectric losses.
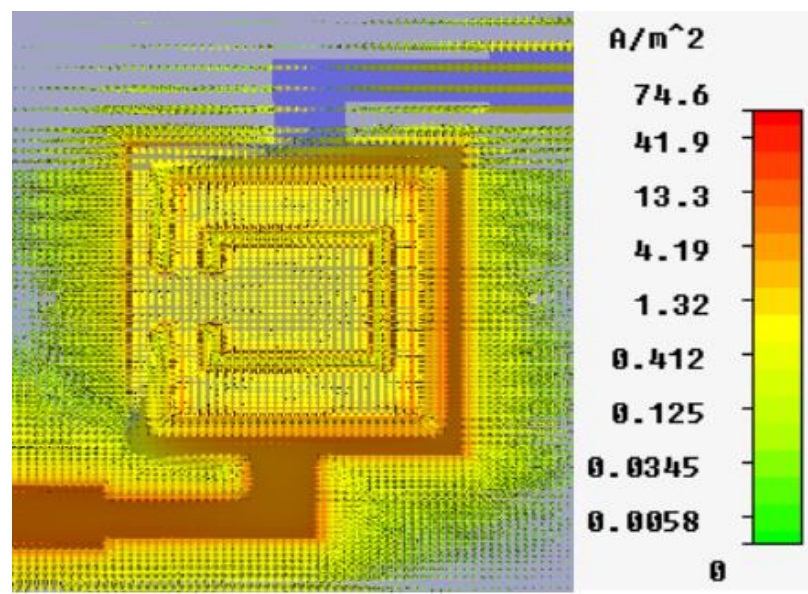

(a)
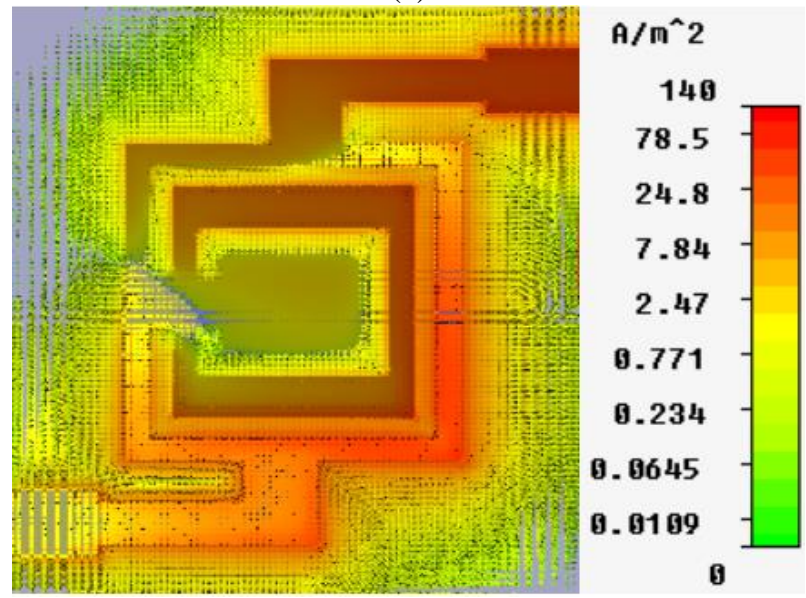

(b)

Figure. 14 (a) Simulated surface current at $2.4 \mathrm{GHz}$ and (b) simulated surface current at $1.7 \mathrm{GHz}$ 


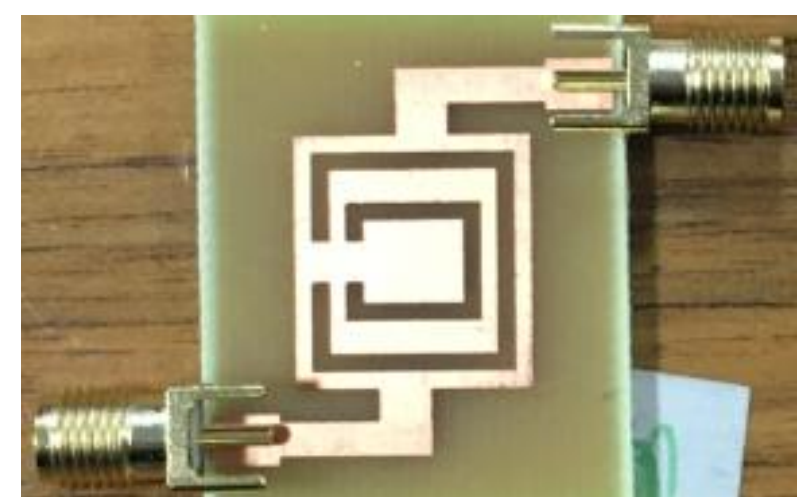

Figure. 15 Simulated surface current at $2.4 \mathrm{GHz}$

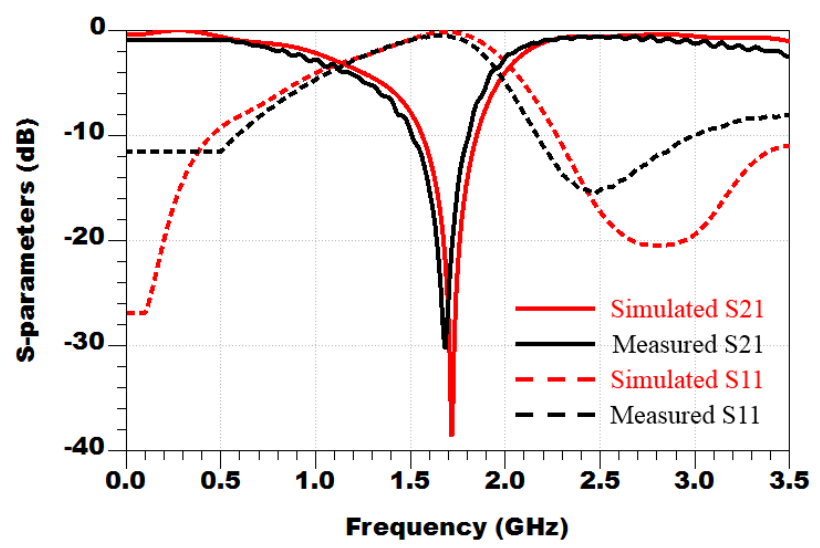

Figure. 16 Simulated and measured S-parameters of the proposed LPF

The fabricated circuit performances are compared with other previously published work in term of overall circuit area, rejected band, attenuation level, and stopband fractional bandwidth FBW that is used to evaluate the unwanted range frequencies of a bandstop filter. As shown in Table 2, we can clearly observe that this filter has remarkably good electrical performances and wide stop-band extended from 1.2 $\mathrm{GHz}$ to $2 \mathrm{GHz}$ Fractional Bandwidth FBW of 50\% and characterized by an excellent deep rejection which can reach more than $30 \mathrm{~dB}$ at the centre frequency. Moreover, the filter has very small and compact size in comparison to its operating frequencies and the circuit area of the previously published papers that are cited in Table 2.

Table 2. Performance comparison among published filters and this work

\begin{tabular}{|c|c|c|c|c|}
\hline $\begin{array}{c}\text { Parameters } \\
\text { /Ref }\end{array}$ & $\begin{array}{c}\text { Rejected } \\
\text { Band(GHz) }\end{array}$ & FBW & $\begin{array}{c}\text { S21 } \\
\text { Deep }\end{array}$ & $\begin{array}{c}\text { Size } \\
\text { mm }^{2}\end{array}$ \\
\hline$[5]$ & {$[1.3-1.7]$} & $26 \%$ & $30 \mathrm{~dB}$ & 1800 \\
\hline$[6]$ & {$[9.19-10.25]$} & $30 \%$ & $30 \mathrm{~dB}$ & 600 \\
\hline$[13]$ & {$[4.4-5.4]$} & $20 \%$ & $25 \mathrm{~dB}$ & $1040 \geq$ \\
\hline$[8]$ & {$[0.975-1.02]$} & $5 \%$ & $35 \mathrm{~dB}$ & 6370 \\
\hline$[7]$ & {$[2.75-3.35]$} & $20 \%$ & $25 \mathrm{~dB}$ & 609 \\
\hline This Work & {$[1.2-2]$} & $50 \%$ & $30 \mathrm{~dB}$ & 576 \\
\hline
\end{tabular}

\section{Conclusion}

A new compact and miniature microstrip bandstop filter based on metamaterial has been suggested in this letter. The stop-band behaviour has been obtained using the complementary split ring resonator unit cell which was etched in the middle top layer of the designed Low-pass structure. It was stimulated optimised using CST Microwave and was fabricated and mounted on Flame Resistant-4 substrate. The proposed circuit has small and compact size moreover a wide stop-band with Fractional Bandwidth FBW of 58\%. This circuit can be considered as a qualified solution for many systems and applications. In our future work, we will focus to improve the first and second pass-bands by using metamaterials on the ground plane of the circuit.

\section{Acknowledgments}

We thank Mr. Mohamed Latrach Professor in ESEO, engineering institute in Angers, France, for allowing us to use all the equipment and solvers available in his laboratory.

\section{References}

[1] H. Shaman, J. S. Hong, "Wideband Bandstop Filter with Cross Coupling", IEEE Transactions on Microwave Theory and Techniques, Vol.55, No.8, pp.1780-1785, 2007.

[2] D. M. Pozar, Microwave Engineering. $3^{\text {rd }}$ Edition, John Wiley \& Sons, 2005

[3] B. Nasiri, A. Errkik. J. Zbitou, A. Tajmouati, L. El Abdellaoui, and M. Latrach, "A Novel Design of a Compact Microstrip Bandstop Filter Based on CSSR", In: Proc. of International Conference on Computing and Wireless Communication Systems, Larache, Morocco, pp.26-30, 2017.

[4] L. Murmu and S. Das, "A Simple Wideband Microstrip Bandstop Filter for WLAN and WiMAX Band Rejection Purpose", In: Proc. of International Conf. On Electrical and Computer Engineering, IEEE, Dhaka, Bangladesh, pp.4749, 2014.

[5] R. Ranjan, A. K. Tiwary, and N. Gupta, "Design and Development of Bandstop Filter Using Spiral Stub", In: Proc. of International Conference on Advances In Computing, Communications and Informatics. IEEE, New Delhi, India, pp. 1360-1365, 2014.

[6] E. Esmaeili and J. Bornemann, "Microstrip Bandstop Filters Using L-and T- Shaped Resonators", In: Proc. of Asia Pacific Microwave Conference. IEEE, Nanjing, China, pp.1-3, 2015. 
[7] B. Nasiri, A. Errkik, J. Zbitou, A. Tajmouati, L. El Abdellaoui, and M. Latrach, "A New Microstrip Band-stop Filter Using Square Split Ring resonator", International Journal Microwave and Optical Technology, Vol.12, No.5, pp.367-373, 2017.

[8] E. J. Naglich and A. C Guyette, "Microwave Bandstop Filters with Minimum through -Line Length", In: Proc. of MTT-S International Microwave Symposium. IEEE, Phoenix, AZ, USA, pp.1-4, 2015.

[9] A. Boutejdar, A. Omar, and E. Burte, "Miniaturized Lowpass and Bandstop Filters Using Controlled Coupling of Open-Loop-Ring Defected Ground Structure (DGS)", Microwave and Optical Technology Letters, Vol.12, No.11, pp.2575-2578, 2010.

[10] A. Boutejdar and A. Omar, "A Miniature 5.2$\mathrm{GHz}$ Bandstop Microstrip Filter Using Multilayer-Technique and Coupled Octagonal Defected Ground Structure", Microwave and Optical Technology Letters, Vol.51, No. 12, pp.2810-2813, 2009.

[11] Y. Zhou and D. L. Su, "A Novel of Wide Band Micro-Strip Filter with Electromagnetic Band Gap Group Structure", In: Proc. of IEEE International Conference on Ultra-Wideband, Nanjing, China, pp1-3, 2010.

[12] A. H. Reja, M. A. Alqaisy, S. N. Ahmed, and A. K. K. A. Raheem, "Design of Metamaterial Stepped-Impedance Microwave LPFs", In: Proc. of World congress on Computer Applications and Information Systems. IEEE, Hammamet, Tunisia, pp. 1-5, 2014.

[13] J. Carver, V. Reignault, and F. Gadot, "Engineering of the Metamaterial-Based CutBand Filter", Applied Physics A, Vol.117, No.2, pp.513-516, 2014.

[14] V. G. Veselago, "The Electrodynamics of Substances with Simultaneous Negative Value of $\varepsilon$ and $\mu$ ", Soviet Physics Uspekhi, Vol.10, No.4, pp.509-514, 1968.

[15] J. B. Pendry, A. J. Holden, and W. J. Stewart, "Extremely Low Frequency Plasmons in Metallic Mesostructures", Phys. Rev. Lett., Vol.76, No.25, pp.4773-4776, 1996.

[16] J. B. Pendry, A. J. Holden, D. J. Robbins, and W. J. Stewart, "Magnetism from Conductors and Enhanced Nonlinear Phenomena", IEEE Transaction on Microwave Theory and Techniques, Vol.47, No.11, pp.2075-2084, 1999.

[17] Y. Yang, Z. Xi, and N. C. Karmakar, "Microstrip Lowpass Filter Based on Split Ring and Complementary Split Ring Resonators",
Microwave and Optical Technology letters., Vol.54, No.7, pp. 1723-1726, 2012.

[18] A. Ennajih, J. Zbitou, M. Latrach, A. Errkik, and R. Mandry, "A New Dual band Printed Metamaterial Antenna for RFID Reader Applications", International Journal of Electrical and Computer Engineering, Vol.7, No.6, pp.3507-3514, 2017.

[19] K. Jang, S. kahng, J. Jeon, and Q. Wu, "Compact Dual-Band Three-Way Metamaterial Power Divider with a Hybrid CRLH Phase-Shift Line", Journal Of Electromagnetic Engineering and Science, Vol.14, No.1, pp.15-24, 2014. 